\title{
Methods Conditional Optimization Dispatching Management Restoration and Modernization Aerial Vehicles
}

\author{
Dmitry O. Krikunov* \\ Military Education and Research Centre of Military-Air Forces \\ "Military-Air Academy \\ Named After Professor N.E. Zhukovsky and Yu.A. Gagarin" \\ 54a Starykh Bolshevikov Str., Voronezh, 394064, Russia
}

Received 03.04.2016, received in revised form 09.02.2017, accepted 21.04.2017

The article considers problem of construction an adequate mathematical model of of dispatching management by reduction of and modernization of aircraft at an aviation repair enterprise. The focus is on the analysis of conditional optimization of dispatching management models for different situations.

Keywords: multiplier, inequality, restrictions, optimization, minimization, function.

Citation: Krikunov D.O. Methods conditional optimization dispatching management restoration and modernization aerial vehicles, J. Sib. Fed. Univ. Eng. technol., 2018, 11(1), 24-28. DOI: 10.17516/1999-494X-0003.

(C) Siberian Federal University. All rights reserved

* Corresponding author E-mail address: krikunofdo@yandex.ru 


\section{Методы условной оптимизации}

\section{диспетчерского управления восстановлением \\ и модернизацией летательных аппаратов}

Д.О. Крикунов

Военный учебно-научный центр Военно-воздушных сил «Военно-воздушная академия имени профессора Н.Е. Жуковского и Ю.А. Гагарина» Россия, 394064, Воронеж, ул. Старых Большевиков, 54 а

В статье рассмотрена задача построения адекватной математической модели диспетчерского управления восстановлением и модернизацией летательных аппаратов на авиационном ремонтном предприятии. Основное внимание уделяется анализу условной оптимизации модели диспетчерского управления для различных ситуаций.

Ключевые слова: множитель, неравенство, ограничения, оптимизация, минимизация, функиия.

Авиаремонтное производство - промышленное производство, продукцией которого является авиационная техника, проходящая капитальный ремонт по достижению ею назначенного ресурса, первого или очередного межремонтного ресурса, капитальный ремонт по тяжелым боевым повреждениям или средний профилактический ремонт. При выполнении ремонта авиационной техники на ремонтном предприятии одновременно производится ее доработка и модернизация. Технологический процесс в авиаремонтном производстве отличается известным единством и состоит из ряда этапов: частичная или полная разборка объекта ремонта, промывка, дефектация, замена или восстановление поврежденных деталей и узлов, замена агрегатов по выработке их ресурсов, сборка и испытания [1].

Таким образом, авиаремонтное производство и организационно, и технологически весьма сложная система.

Построение адекватной математической модели такой системы позволит выбрать её оптимальную структуру, оптимизирующую загрузку отдельных компонентов с использованием различных критериев.

Ряд инженерных задач связан с оптимизацией производства при наличии некоторого количества ограничений на управляемые переменные. Такие ограничения существенно уменьшают размеры области, в которой ищут оптимум. Рассмотрим необходимые и достаточные условия оптимума в задачах с ограничениями, которые иначе называют задачами условной оптимизации [1].

Так, существует задача с ограничениями в виде равенства: $f(x) \rightarrow \min , x \in R^{n}$ при ограничениях $h_{k}=0, k=1,2, \ldots, K$.

Одним из методов ее решения является метод множителей Лагранжа.

С помощью метода множителей Лагранжа устанавливаются необходимые условия, позволяющие идентифицировать точки оптимума в задачах оптимизации с ограничениямиравенствами. При этом задача с ограничениями преобразуется в эквивалентную задачу безу- 
словной оптимизации, в которой фигурируют некоторые неизвестные параметры, называемые множителями Лагранжа.

Рассмотрим задачу с одним ограничением-неравенством:

$$
\begin{aligned}
& f(x) \rightarrow \min , x \in R^{n}, \\
& h_{l}(x)=0 .
\end{aligned}
$$

В соответствии с методом множителей Лагранжа эта задача преобразуется в следующую задачу безусловной минимизации:

$$
L(x ; \lambda)=f(x)-\lambda h_{1}(x) \rightarrow \min , x \in R^{n} .
$$

Функция $L(x ; \lambda)$ называется функцией Лагранжа. Здесь $\lambda$ - множитель Лагранжа.

Тогда при заданном значении $\lambda=\lambda^{0}$ безусловный минимум функции $L(x ; \lambda)$ по переменной $x$ достигается в точке $x=x^{0}$ и $x^{0}$ удовлетворяет уравнению $h_{1}\left(x^{0}\right)=0$.

Необходимо подобрать значение $\lambda=\lambda^{0}$ таким образом, чтобы координата точки безусловного минимума $x^{0}$ удовлетворяла равенству (2). Это можно сделать, если, рассматривая $\lambda$ как переменную, найти безусловный минимум функции Лагранжа (3) в виде функции $\lambda$, а затем выбрать значение $\lambda$, при котором выполняется равенство (2).

Метод множителей Лагранжа можно распространить на случай, когда задача имеет несколько ограничений в виде равенств:

$$
\begin{aligned}
& f(x) \rightarrow \min , x \in R^{n}, \\
& h_{k}(x)=0, k=1,2, \ldots, K
\end{aligned}
$$

Функция Лагранжа принимает вид $L(x ; \lambda)=f(x)-\sum_{k=1}^{K} \lambda_{k} h_{k}$.

В этом случае $\lambda_{1}, \ldots, \lambda_{K}-$ множители Лагранжа, т.е. неизвестные параметры, значения которых нужно определить [2]. Приравнивая частные производные $L$ по $x$ нулю, получаем следующую систему:

$$
\frac{\partial L(x, \lambda)}{\partial x_{1}}=\frac{\partial L(x, \lambda)}{\partial x_{2}}=\cdots=\frac{\partial L(x, \lambda)}{\partial x_{n}}=0 .
$$

Если найти решение этой системы в виде функций от вектора $\lambda$ затруднительно, то можно расширить последнюю систему путем включения в нее ограничений-равенств:

$$
h_{1}(x)=0, h_{2}(x)=0, \ldots, h_{k}(x)=0 .
$$

Решение расширенной системы, состоящей из $N+K$ уравнений с $N+K$ неизвестными, определяет стационарную точку функции $L$. Затем реализуется процедура проверки на минимум или максимум, которая проводится на основе вычисления элементов матрицы Гессе функции Лагранжа, рассматриваемой как функция от $x$.

Рассмотрим задачу с ограничениями в виде неравенств:

$$
f(x) \rightarrow \min , x \in R^{n},
$$


с ограничениями-неравенствами:

$$
g_{j}(x) \leq 0, j=1,2, \ldots, J
$$

Пусть область (9) (обозначим ее $D$ ) - не пустое, ограниченное замкнутое множество. Функция Лагранжа для задачи (8) с ограничениями (9) определяется формулой

$$
L(x ; \lambda)=f(x)-\sum_{j=1}^{J} \lambda_{j} g_{j}(x)=f(x)-\lambda^{T} g(x),
$$

где $\lambda$ - вектор множителей Лагранжа: $\lambda=\left(\lambda_{1}, \ldots, \lambda_{j}\right)^{T}, g=\left(g_{1}, \ldots, g_{j}\right)^{T}$.

В точке локального минимума задачи (8), (9) каждое из ограничений (9) выполняется либо в виде равенства $g_{j}\left(x^{*}\right)=0$, либо в виде неравенства $g_{j}\left(x^{*}\right)<0$. Ограничения первого вида называются активными ограничениями. Остальные ограничения - неактивные ограничения.

Если точка $x^{*} \in D$ и ограничения $g_{j k}\left(x^{*}\right) \leq 0, j_{k}=1, \ldots, s, s \leq J$ активны, то условие линейной независимости градиентов функций $g_{j k}\left(x^{*}\right), j_{k}=1, \ldots, s, s \leq J$ активных ограничений в точке $x^{*}$ называется условием регулярности ограничивающих функций в точке $x^{*}$. Это условие означает, что, например, при $n=2$ количество ограничивающих функций, проходящих через точку $x^{*}$, не должно превышать 2 и в точке $x^{*}$ векторы $\nabla g_{1}(x), \nabla g_{2}(x)$ не должны быть коллинеарны [3].

Большое значение в теории и вычислительной практике имеет следующая теорема КунаТаккера для задачи условной оптимизации с ограничениями типа неравенств.

Пусть функция $f(x)$ и функция $g_{j}\left(x^{*}\right) \leq 0, j=1,2, \ldots, J$ имеют непрерывные частные производные в некоторой окрестности точки $x^{*}$, и пусть эта точка является точкой локального минимума функции $f(x)$ при ограничениях $g_{j}\left(x^{*}\right) \leq 0$, удовлетворяющих в точке $x^{*}$ условию регулярности ограничивающих функций. Тогда существуют такие неотрицательные множители Лагранжа $\lambda_{1}, \ldots, \lambda_{J}$, что для функции Лагранжа $L(x ; \lambda)$ точка $x^{*}$ является стационарной точкой, т.e. $\nabla_{x} L\left(x^{*} ; \lambda\right)=\nabla f\left(x^{*}\right)-\sum_{j=1}^{J} \lambda_{j} \nabla g_{j}\left(x^{*}\right)=0$.

Своеобразным и очень эффективным методом оптимизации признан метод факторов (или множителей), который основан на санкции типа «квадрат срезки» для ограничений-неравенств [2].

Такая санкция определяется следующим образом:

$$
S=R\langle g(x)\rangle^{2}
$$

где срезка $t$ определяется так:

$$
\langle t\rangle=\left\{\begin{array}{l}
t, \text { если } t \geq 0, \\
0, \text { если } t<0 .
\end{array}\right.
$$

Эта санкция внешняя и стационарные точки функции $\mathrm{Q}(\mathrm{x}, \mathrm{R})$ могут оказаться недопустимыми. С другой стороны, недопустимые точки не создают в данном случае дополнительных сложностей по сравнению с допустимыми. Различие между ними состоит лишь в том, что в допустимых точках санкция равна нулю.

В методе факторов на каждой итерации производится безусловная минимизация функции 


$$
Q(x, \sigma, \tau)=f(x)+R \sum_{j=1}^{J}\left\{\left\langle g_{j}(x)+\sigma_{j}\right\rangle^{2}-\sigma_{j}^{2}\right\}+R \sum_{k=1}^{K}\left\{\left[h_{k}(x)+\tau_{k}\right]^{2}+\tau_{k}^{2}\right\},
$$

где $R$ - постоянный весовой коэффициент, а угловые скобки обозначают операцию срезки. Параметры (факторы) $\sigma_{j}$ и $\tau_{k}$ осуществляют сдвиг санкционных слагаемых. Компоненты векторов $\sigma$ и $\tau$ меняются по ходу вычислений, однако в процессе решения каждой вспомогательной безусловной задачи оба эти вектора остаются постоянными. Начальные значения факторов $\sigma$ и $\tau$ можно выбрать нулевыми. Обозначим через $x^{m}$ точку минимума функции $Q\left(x, \sigma^{m}, \tau^{m}\right)$, используемой на $m$-й итерации.

При переходе к $(m+1)-$ й итерации факторы пересчитываются по формулам

$$
\begin{gathered}
\sigma_{j}^{m+1}=\left\langle g_{j}\left(x^{m}\right)+\sigma_{j}^{m}\right\rangle, \mathrm{j}=1, \ldots, \mathrm{J}, \\
\tau_{j}^{m+1}=h_{k}\left(x^{m}\right)+\tau_{k}^{m}, \mathrm{k}=1, \ldots, \mathrm{K} .
\end{gathered}
$$

Формулы пересчета таковы, что в результате сдвига при переходе к новой подзадаче санкции за нарушение ограничений возрастают, и вследствие этого точки $x^{m}$ приближаются к допустимой области.

Для контроля сходимости метода используют последовательности $x^{m}, \sigma^{m}, \tau^{m}, f\left(x^{m}\right), g\left(x^{m}\right)$, $h\left(x^{m}\right)$. Прекращение основного процесса происходит, когда члены по крайней мере одной из этих последовательностей перестают изменяться при пересчете факторов и последующей безусловной минимизации. Заметим, что величина положительного параметра $R$ влияет на свойства метода, но конструктивного алгоритма его выбора не существует.

Таким образом, решая задачу с ограничениями в виде равенств с использованием метода множителей Лагранжа, мы преобразуем задачу с ограничениями в задачу безусловной оптимизации. Кроме того, в автоматизированной системе управления важное значение может иметь решение задачи условной оптимизации с ограничениями типа неравенств с применением теоремы Куна-Таккера.

Построение адекватной математической модели для оптимизации загрузки системы диспетчерского управления восстановлением и модернизацией летательных аппаратов на авиационном ремонтном предприятии может быть осуществлено как комплекс задач с ограничениями в виде равенства и в виде неравенств [1].

\section{Список литературы}

[1] Васецкий В.В., Ирхин В.П. Автоматизация диспетчерского управления авиачионного ремонтного предприятия. Воронеж: ВАИУ, 2011. 206 c. [Vaseckij V.V., Irhin V.P. Automation of the dispatch control of an aircraft repair enterprise. Voronezh, VAIU, 2011, 206 p. (in Russian)]

[2] Клир Дж. Системология. Автоматизаиия решения системных задач: Пер. с англ. М.: Радио и связь, 1990. 544 с. [Klir Dzh. Systemology. Automation of the solution of system tasks. Moskva, Radio i svjaz', 1990, 544 p. (in Russian)]

[3] Есипов Б.А. Методы оптимизации и исследование операций: Конспект лекций. Самара: Изд-во Самар. гос. аэрокосм. ун-та, 2007. 180 с. [Esipov B.A. Optimization methods and operations research. Samara, SGAU, 2007, 180 p. (in Russian)]

$$
-28-
$$

